\title{
Un «clásico tímido». Giorgio Bassani in Spagna: dalla Transizione democratica a oggi
}

Un «clásico tímido». Giorgio Bassani en España desde la Transición Democrática hasta hoy

Un « clásico tímido ». Giorgio Bassani en Espagne de la Transition démocratique à aujourd'hui

\section{Silvia Datteroni}

\section{OpenEdition Journals}

Edizione digitale

URL: http://journals.openedition.org/cei/3798

DOI: 10.4000/cei.3798

ISSN: 2260-779X

Editore

UGA Éditions/Université Grenoble Alpes

\section{Edizione cartacea}

ISBN: 978-2-37747-040-2

ISSN: 1770-9571

Notizia bibliografica digitale

Silvia Datteroni, «Un «clásico tímido». Giorgio Bassani in Spagna: dalla Transizione democratica a oggi», Cahiers d'études italiennes [Online], 26 | 2018, online dal 28 février 2018, consultato il 27 mars 2021. URL: http://journals.openedition.org/cei/3798 ; DOI: https://doi.org/10.4000/cei.3798

Questo documento è stato generato automaticamente il 27 mars 2021

(c) ELLUG 


\section{Un «clásico tímido». Giorgio Bassani in Spagna: dalla Transizione democratica a oggi}

Un «clásico tímido». Giorgio Bassani en España desde la Transición Democrática hasta hoy

Un « clásico tímido ». Giorgio Bassani en Espagne de la Transition démocratique à aujourd'hui

Silvia Datteroni

\section{Osservazioni preliminari: Giorgio Bassani scrittore «einaudito» sotto il franchismo}

1 L'avventura editoriale di Giorgio Bassani in Spagna inizia negli anni ' $60^{1}$, quando l'editore catalano Carlos Barral decide di pubblicare l'opera narrativa del ferrarese con il marchio di famiglia Seix-Barral. La corrispondenza epistolare tra l'editore spagnolo e l'agente letterario di Bassani, Erich Linder, conservata presso la Fondazione Arnoldo e Alberto Mondadori, ha permesso di ricostruire le tappe principali della vicenda editoriale dello scrittore nella Spagna franchista, orientando una prima riflessione sulla ricezione di Bassani durante la dittatura.

Le poche carte a disposizione permettono una lettura lacunosa della vicenda, ma bastano a dimostrare che l'interesse per l'opera di Bassani nasce all'inizio degli anni ' $60^{2}$ e si mantiene approssimativamente per tutto il periodo 1963-1974. La prima opera di Bassani a essere pubblicata in Spagna è Eljardín de los Finzi-Contini, uscito nel 1963, e poi ristampato più volte negli anni dalla stessa casa editrice: nel 1968 - quando Barral riceve un'autorizzazione 'extraoficial' dello Stato a pubblicare libri in formato economico - e poi di nuovo nel '73 e nel '76. Sempre per i tipi di Seix Barral, si pubblica nel 1967 il volume Historias de Ferrara e nel 1969 si dà alle stampe Detrás de la puerta, che nel 1964 era stato rifiutato dalla censura. Nel 1968 esce L'Airone (La garza), 
poi ristampato nel 1970. Infine nel 1974 Seix-Barral pubblica L'odore del fieno (El olor del heno). Nel frattempo, Carlos Barral lascia la casa editrice e nel 1970 fonda Barral Editores. Con il nuovo marchio editoriale, nel 1971, egli pubblica nuovamente le storie ferraresi con il titolo Lida Mantovani y otras historias de Ferrara e nel 1972 Gli occhiali d'oro (Los anteojos de oro) nella collezione tascabile «De bolsillo».

Da quanto precede si può dedurre che, nonostante le molteplici pressioni esercitate dalla Oficina de orientación bibliográfica - un organismo che dipendeva dal Ministerio de Información y Turismo, e più comunemente conosciuto come 'censura' - l'opera del ferrarese ha potuto filtrare in Spagna durante tutto il periodo franchista grazie all'attività di Seix-Barral, una casa editrice che ha svolto un ruolo importante durante tutti quegli anni difficili. Le ricerche compiute recentemente da Bresadola ${ }^{3}$ presso l'Archivio di Stato di Alcalá de Henares (Madrid), dimostrano che la censura non è intervenuta troppo severamente sull'opera di Bassani, e non ne ha impedito la pubblicazione, a eccezione di Dietro la porta, inizialmente proibito, ma autorizzato a distanza di alcuni anni. Si può inoltre constatare che gli interventi della censura sono determinati sia da considerazioni 'morali', relative al contenuto dell'opera, sia da dinamiche extra-letterarie; fra queste ultime è da segnalare in particolare l'avversione nei confronti dell'editore italiano di Bassani, Giulio Einaudi, considerato come un soggetto pericoloso dal regime franchista e dichiarato 'persona non grata' in Spagna. Einaudi aveva infatti solidarizzato con la causa antifranchista pubblicando nel 1962 un'antologia dal titolo Canti della Nuova Resistenza spagnola: 1936-1961, bollata dal regime franchista come un atto di "propaganda sovversiva al servizio dei comunisti» ${ }^{4}$. L'ostilità verso Einaudi, aggravata dalla sua partecipazione agli Encuentros Formentor come editore engagé, non favorì Bassani, presto identificato come autore einaudiano, o «einaudito» ${ }^{5}$.

4 È dunque probabile che l'intervento della censura, volto soprattutto a colpire Einaudi, abbia rallentato la ricezione dello scrittore in Spagna; di converso è possibile che l'ammirazione di Barral per l'editore torinese - da lui definito un vero "maestro» 6 abbia contribuito, almeno inizialmente, al suo interesse per Bassani.

Durante l'epoca franchista, caratterizzata da una retorica dittatoriale asfissiante e da un controllo assoluto sulla vita culturale del Paese, Giorgio Bassani, come tanti altri scrittori suoi contemporanei, è pubblicato senza troppo successo di critica. Il periodico «La Vanguardia» di Barcellona gli dedica brevi e puntuali articoli, che coincidono per la maggior parte con l'uscita dei suoi libri. Vi si leggono accenni al linguaggio e allo stile dello scrittore, ma non ancora una riflessione sulla sua poetica. Nel 1963, per esempio, l'uscita del Giardino è annunciata in un trafiletto dedicato alle pubblicazioni di SeixBarral, che menziona la fortuna del libro in Italia e in Francia e la sua «indiscutible calidad» ${ }^{7}$. Nel dicembre dello stesso anno un articolo del già ricordato Juan Ramón Masoliver è una sorta di recensione al Giardino dei Finzi-Contini. Il critico, che fa riferimento al tono lirico della narrazione e ai ripetuti contatti tra prosa e poesia, sottolinea l'importanza, in Bassani, dei dettagli che «constituyen la atmósfera» $\mathrm{e}$ rendono l'istante eterno ${ }^{8}$.

6 L'attenzione critica verso Bassani crescerà con gli anni. Nel decennio '60-'70, in una classe intellettuale minoritaria se non clandestina prevaleva l'urgenza di colmare un allarmante ritardo culturale, dovuto al franchismo; di conseguenza, le esperienze straniere che erano inserite nel tessuto culturale spagnolo erano riunite sotto etichette generiche, senza che ne fosse pienamente assimilata la specificità. Al suo ingresso nella 
Spagna di Franco lo scrittore ferrarese non è chiaramente distinto dal neorealismo ${ }^{9}$. Lo storicismo di Bassani, che scandaglia la storia senza semplificazioni critiche né giudizi stereotipati sui personaggi, è assai lontana dall'ormai agonizzante realismo social ${ }^{10}$ spagnolo, ma solo in un secondo momento sarà riconosciuto come un esempio idealmente utile dal gruppo intellettuale militante dei letraheridos ${ }^{11}$.

7 La scrittura memoriale di Bassani, concepita dallo scrittore come un dovere artistico e morale, come un'arma per stanare le contraddizioni di una società postbellica che rifugge dalle proprie responsabilità storiche, sembra offrire un'alternativa alla letteratura spagnola di quegli anni, una via per trattare in modo nuovo una ferita storica e nazionale ancora aperta, generata dalla guerra civile e dalla dittatura. Il socialrealismo degli spagnoli, rappresentato da scrittori come Camilo José Cela, Ignacio Aldecoa, Rafael Sánchez Ferlosio, Juan Marsé, Juan Goytisolo e altri si mostrava infatti incapace di penetrare le contraddizioni di una società ancora divisa ideologicamente, $\mathrm{e}$ generava un'aporia stilistica che era ormai oggetto di importanti dibattiti culturali. Senza poter asserire con sicurezza un'influenza diretta di Bassani sugli scrittori spagnoli dell'epoca, si può comunque avanzare l'ipotesi di una preoccupazione estetica condivisa $^{12}$, che condurrà gli intellettuali della penisola iberica a un realismo dialéctico - la cui apparizione si fa in genere coincidere con la pubblicazione, nel 1962, di Tiempos de silencio di Luís Martín-Santos - che a sua volta preparerà il terreno a nuove esperienze letterarie della transizione democratica.

\section{Anni Settanta e Ottanta: «ll romanzo di Ferrara» in Spagna tra boom editoriale, impegno civile e poetica d'autore}

8 Il periodo post-franchista - conosciuto storicamente con l'emblematica etichetta di Transizione $^{13}$ - è una fase di assestamento politico e sociale, caratterizzata culturalmente dalla fine del realismo social.

Durante questa fase di ricostruzione nel segno della continuità, in cui la Spagna prova a rifondare una propria identità politica e culturale, abbandonando la mimesis impostata ideologicamente della realtà sociale ${ }^{14} \mathrm{e}$ aprendosi sempre più a influenze occidentali, non si parla molto di Giorgio Bassani. Il suo realismo lirico, così atipico da renderlo un caso a parte sia in Italia che in Spagna, non sembra trovare spazio all'interno di un'epoca satura di trasformazioni, in cui lo sperimentalismo prende campo con il modello indiscusso di Juan Benet.

10 Tuttavia, le frenesie sperimentaliste della Spagna della Transizione, che portano alla pratica di modelli metanarrativi e antinarrativi rappresentati da autori come Enrique Vila-Matas, Juan Goytisolo, Juan Benet e Julián Ríos, non riescono a liquidare completamente l'estetica realista, legata al bisogno umano e sociale di capire un passato prossimo non ancora veramente archiviato. Senza tornare al socialrealismo si sviluppa quindi, parallelamente al metaromanzo e all'antiromanzo, un tipo di letteratura 'utile', in grado cioè di interpretare le preoccupazioni contemporanee, sopraggiunte con la democrazia. Torna la narratività, e con essa memorie, diari, autobiografie e romanzi storici. Il genere cronachistico e saggistico torna in auge, supportato anche dalla politica editoriale delle principali case editrici, che si interrogano sul genere e sugli autori su cui scommettere. 

storica. E capire in che modo il paradigma della memoria abbia influito in un'epoca di bilanci e analisi delle responsabilità, nonché sulla successiva ricostruzione dell'identità nazionale, è indispensabile se si vuole comprendere la 'funzione Bassani' nella Spagna post-franchista. nella collana Hispánica Nova e con il nuovo marchio Barral Editores. Quella di Bassani è una presenza forse anomala nel catalogo barraliano, soprattutto se consideriamo la traiettoria editoriale dello spagnolo: dopo il divorzio dalla casa editrice familiare, la leggendaria Seix Barral, in cui aveva ideato la collana Biblioteca Breve, responsabile della promozione letteraria del socialrealismo prima e degli scrittori del boom latinoamericano poi, Carlos Barral vira di bordo e scommette su istanze sperimentali, dovendo però scendere a compromessi e pubblicare parallelamente libri di stampo enciclopedico e scientifico che costituiscono il grosso del fatturato della nuova Casa. La presenza di un classico come Bassani può spiegarsi come un'eccezione nella linea editoriale del catalogo, proprio in virtù di quel suo realismo eterodosso che continua a non trovare un equivalente nella Spagna del momento e il cui valore canonico garantisce prestigio al catalogo di un editore «exquisito» ${ }^{15}$.

Tuttavia, durante i primi anni della Transizione, Bassani è presente forse solo per un fenomeno di inerzia editoriale. Nei cataloghi troviamo solo la riedizione del Giardino nel 1976, probabilmente legata alla precedente uscita nelle sale spagnole dell'omonimo film di De Sica, nel 1971. È probabile che la mediazione cinematografica dell'opera di Bassani abbia contribuito alla ricezione dello scrittore nella penisola iberica, mantenendo un certo interesse nei suoi confronti anche in quei periodi amorfi in cui la critica allontanava lo sguardo dalla sua opera.

14 Solo negli anni '80, dopo che l'autore sistema la propria opera e licenzia presso Mondadori il complesso e definitivo ciclo del Romanzo di Ferrara, Bassani torna con forza sulle pagine dei principali quotidiani e delle riviste specializzate.

Tra il 1981 e il 1985, Bassani si reca più volte in Spagna; la prima tappa documentata è una visita all'Istituto italiano di cultura di Madrid, dove una conferenza permette all'autore di presentare il Romanzo di Ferrara e di chiarire la sua poetica. Lo scrittore insiste sul valore politico-resistenziale dell'opera, in particolare del Giardino dei FinziContini, che definisce come «toma de posición contra el fascismo». Si tratta di un tema sensibile per una Spagna martoriata da quarant'anni di dittatura e con un'esperienza underground minima ed elitaria alle spalle ${ }^{16}$. Nell'articolo di José $\mathrm{F}$. Beaumont che annuncia la visita madrilena dello scrittore, sono trascritte alcune dichiarazioni rilasciate da Bassani a proposito del suo orientamento politico vicino ai «radicali italiani della democrazia di sinistra, laico, non marxista e con una forte esperienza antifascista $\aleph^{17}$. Beaumont fa poi uno dei primi accenni alla poetica post-neorealista di Bassani e alla struttura del Romanzo di Ferrara, definito come «l'opera di un artista a cui interessa solamente essere artista, senza per questo smettere di essere autentico, senza allontanarsi dalla verità».

16 Nello stesso anno, forse proprio in occasione dell'invito all'Istituto italiano di cultura di Madrid, Bassani compare come ospite di una puntata del programma televisivo Encuentro con las letras. Si tratta di un programma nato nel 1976, nello stesso anno in cui sono fondati i quotidiani «El País» ${ }^{18}$, «Diario 16» (1976-2001) e la rivista specializzata «Historia 16» (1976-2008), tutti mezzi di informazione che avranno un ruolo di primo 
piano durante la transizione spagnola. Il 1981 è l'ultimo anno di emissione del programma diretto da Roberto Vélez ed è caratterizzato da una decisa apertura alle lettere straniere. È proprio in quest'ultima fase 'aperturista' che Esther Benítez e José Luís Jover intervistano Giorgio Bassani e Leonardo Sciascia ${ }^{19}$.

È degno di rilievo che la 'riscoperta' di Bassani da parte di un settore della critica specializzata avvenga in un momento di trasformazione degli abiti e dei costumi sociali, in cui i valori di riferimento diventano cosmopolitismo, trasgressione, ambiguità sessuale, ecc., tutti aspetti della movida madrileña immortalati da Almodóvar nei suoi film diventati ormai cult. Ed è altrettanto significativo che in quegli anni euforici lo slancio culturale extra-nazionale avvenga proprio in direzione del realismo di Giorgio Bassani.

Nel 1983 Bassani è di nuovo in Spagna, a Valencia, ospite dell'Encuentro de Escritores del Mediterráneo. Si tratta di un incontro che propone una riflessione critica sul passato e sulla memoria, e al quale prendono parte più di duecento intellettuali, tra cui Octavio Paz, che inaugura l'evento. Si tratta in sostanza di una commemorazione di un precedente incontro, svoltosi cinquant'anni prima in piena Guerra Civile, nella stessa Valencia. Bassani è presente con Carlos Barral, Francisco Ayala, Heberto Padilla, Francisco Rico, Jaime Salinas, Rosa Cachel e altri, ed è intervistato dallo scrittore Antonio Colinas, che ha da poco vinto il Premio Reína Sofia de Poesía Iberoamericana. Questa è l'unica intervista documentata di quegli anni in cui Bassani, partendo da tematiche morali universali, e riflettendo in particolare sui problemi cui deve far fronte uno scrittore dell'area mediterranea, approda a una dichiarazione di poetica in cui spiega il suo peculiare cortocircuito tra i generi, il suo altrettanto peculiare processo variantistico, che lo porta nel corso degli anni a una continua revisione e correzione dei testi, e il suo attivismo ambientalista, che compare tra le righe di una feroce critica della disumanizzazione dell'arte come conseguenza dell'industrialismo ${ }^{20}$. In un passo di particolare interesse, Bassani spiega che il compito di uno scrittore è quello di far capire come è «necessario essere», evitando ogni soluzione astratta. Secondo lui Il Romanzo di Ferrara è «una respuesta» alla società di oggi:

Mi importa che sia conosciuto fuori d'Italia e, soprattutto che sia conosciuto [...] nell'ambito della lingua spagnola. Uscirà presto in Germania. Nell'America del Nord non sono sicuro che si riuscirà a pubblicarlo, perché lì non digeriscono opere di questo tipo. Se c'è un libro lontano dallo spirito della civiltà industriale è il mio.

Dopo alcuni chiarimenti dello scrittore sul ciclo narrativo ferrarese, Colinas domanda a Bassani le sue impressioni sul mondo attuale. La sua risposta racchiude in poche frasi informali le sue convinzioni più profonde:

È difficile che un poeta possa fare qualcosa per opporsi alla cosificazione del mondo. Può solamente mostrare la sua opposizione essendo se stesso e solo se stesso. Il nostro è un tempo tragico, che ha preferito l'industrialismo all'umanità. La prima e la seconda guerra mondiale (e, concretamente, il nazismo) sono il risultato, il segno di questa crisi, di questa direzione che ha preso l'industrializzazione. Crisi profonda, tremenda, che ci può portare alla fine del mondo. Noi abbiamo vissuto tutta questa crisi attraverso il fascismo. Per questo siamo già vaccinati, sappiamo che, in parte, abbiamo arricchito la nostra esperienza. In sostanza, il mondo ha bisogno di poeti e molto meno di fabbricatori. Finché ci saranno poeti e scrittori, il mondo non sarà definitivamente perduto e ci sarà speranza. Non dobbiamo arrenderci a coloro che vogliono che anche i poeti si trasformino in cose. Certa letteratura di consumo non entra, chiaramente, in questo schema. 


\section{ricorda come un uomo dallo sguardo nostalgico e profondamente coerente con il} proprio impegno storico-poetico. rilasciate da Bassani durante un seminario su «Trayectos narrativos y urbanos en el siglo XX», organizzato dall'Università Internazionale Menéndez Pelayo di Barcellona nel 1984 ${ }^{21}$. Sappiamo da Munné che durante il seminario Bassani parlò del Gattopardo di Giuseppe Tomasi di Lampedusa, la cui scoperta e successiva pubblicazione nel 1958 - in Spagna si pubblicherà nel 1984 per i tipi Seix Barral nella traduzione di Fernando Gutiérrez - avevano reso lo scrittore molto noto in Spagna, tanto da essere identificato talvolta con l'etichetta di «el descubridor de El Gatopardo»" ${ }^{22}$.

Ancora nel 1985, nel giornale barcellonese «La Vanguardia», lo scrittore Robert Saladrigas dedica una rassegna critica completa all'opera di Bassani ${ }^{23}$, in occasione dell'allora recente pubblicazione della traduzione spagnola del Romanzo di Ferrara, uscita nel 1984 per i tipi di Bruguera ${ }^{24}$. Saladrigas ne sottolinea le doti di attento «miniaturista» della struttura narrativa e lo allontana dal costumbrismo ${ }^{25}$ di alcuni scrittori italiani coevi, tra cui Vasco Pratolini. Scrive inoltre che Bassani «contrappone la dolcezza del paesaggio emiliano all'immagine desolata della condizione umana, incapsulata nella solitudine dei vinti», e mette l'accento su ciò che definisce come un ammirevole processo evocativo, in cui l'impulso poetico prevale sul flusso realista. Lo scrittore spagnolo sarà uno dei primi a rilevare la profonda capacità di testimonianza dell'opera del ferrarese, vissuta quasi come un progetto di vita, sul quale altri critici attenti e sensibili alla tematica della memoria si soffermeranno in seguito. Fin dagli anni ' 80 , un motivo ricorrente nella critica è la singolarità di Bassani, il fatto che lo scrittore sia un caso letterario non facilmente inquadrabile né in Spagna né in Italia. È così cresciuta l'attenzione degli intellettuali spagnoli per un autore riscoperto anche a destiempo, forse proprio in virtù della sua poetica atemporale che si adatta a vecchi $\mathrm{e}$ nuovi, traballanti orizzonti culturali della penisola iberica.

\section{L'esercizio di memoria degli anni Novanta come chiave interpretativa della 'funzione Bassani' nella Spagna del $\mathrm{XXI}$ secolo}

La prudenza con cui negli anni ' 80 si analizzano in Spagna colpe e responsabilità storiche del passato, porterà a uno strappo generazionale insanabile di cui sarà permeato anche il mondo della cultura. Il patto di silenzio fra i diversi partiti politici aveva quasi prodotto un'amnesia storica, e allontanato i narradores novísimos ${ }^{26}$ dalle generazioni passate.

Nei primi anni Novanta si produce invece un'inversione di tendenza e si inizia così a rivendicare la memoria storica. Il passato allora riaffiora intenzionalmente, progressivamente, come strumento critico, e finisce per diventare lo scenario privilegiato di una ricostruzione identitaria del Paese.

Si spiegano in questo modo alcuni saggi degli anni zero, tutti successivi alla morte dello scrittore ferrarese e legati alla gestazione di un nuovo paradigma politico della memoria $^{27}$. Lo scrittore Alejandro Gándara ${ }^{28}$ si sofferma per esempio sul carattere atemporale della scrittura bassaniana, che sostiene quasi per contrasto l'ossatura di

Cahiers d'études italiennes, 26 | 2018 
una composizione narrativa che consegna alla memoria storica le micro-storie degli abitanti di Ferrara. Javier Aparicio Maydeu parla invece di una «memoria ficcional»" ${ }^{29}$, risultato di oculate scelte stilistiche che rendono possibile un trattamento linguistico del dolore esistenziale e della solitudine attraverso lo spazio metafisico di un sommesso simbolismo e di una memoria affettiva. Secondo Aparicio Maydeu la scrittura abile e precisa di Bassani conferisce all'opera una 'missione evocatrice' che riesce a prolungare nel tempo una storia bloccata tra immaginazione e memoria. Mario Goloboff approfondisce invece la 'funzione Ferrara' nell'opera di Bassani e parla di un'evidente e volontaria commistione di racconto e testimonianza, risultato di una «función esencial» concessa alla memoria, all'atto di ricordare [rememoración], inteso come punto di partenza per la costruzione letteraria ${ }^{30}$.

Il poeta Luís Mateo Díez, uno dei novísimos narradores, inserisce Bassani in una «irrepetible generación ${ }^{31}$ di romanzieri italiani e Joaquín Torán - in un articolo dal titolo di forte valore simbolico, in quanto stabilisce un'equivalenza fra il nome dello scrittore e un'esigenza politico-culturale di dimensioni europee ${ }^{32}-$ lo colloca all'interno di un «ejercito de renovación» di cui fanno parte anche Calvino, Ginzburg, Pavese e Vittorini, tutti autori con "pedigrí antifascista». La sua sorprendente capacità di 'sentire' la vita senza svelarne il mistero è vincolata secondo Torán a una poetica del sentimento elegiaco e a un potere evocativo della memoria che rielabora i ricordi in chiave intimistica. Per il poeta spagnolo, l'aver prodotto un discorso sui «giorni di allora» [«los días de entonces»] rappresenta molto più di un «ejercicio de responsabilidad» verso se stesso e le proprie origini. Scrive in particolare Torán:

In un momento (il duro dopoguerra) e in un luogo (l'Italia) in cui cineasti e scrittori concentravano i loro sguardi sull'incerto presente, Giorgio Bassani [...] guardava inesorabilmente indietro. Mentre il Paese cercava di rinnegare i suoi peccati fascisti, lui sentiva l'imperiosa necessità di non dimenticare.

Ciò che più colpisce di Bassani, in una Spagna sospesa tra la necessità di riscattare il passato e la scarsa capacità di farlo, è l'atteggiamento di tutore della memoria: Bassani non vuole dimenticare e agli spagnoli non importa tanto che cosa lui non voglia dimenticare ma perché non vuole e come ci riesce. Dalla scrittura di Bassani si può imparare a far luce sugli angoli più scuri della storia nazionale e il microcosmo ferrarese diventa allora un sorta di correlativo oggettivo della macro-storia postfranchista. In un articolo del 2009, España e Israel: ¿Dónde está Micol? ${ }^{33}$, Enric Juliana, codirettore de «La Vanguardia» di Barcellona, riflette sull'atipicità dell'atteggiamento degli spagnoli nei confronti della questione ebraica. Secondo Juliana la Spagna è l'unico Paese occidentale a non aver fatto mea culpa per l'olocausto, a non aver sentito la necessità di comprendere l'accaduto, e questo a causa di un scarso sentimento di empatia per uno sterminio che non ha vissuto in situ. All'epoca delle leggi razziali, non c'erano ebrei in Spagna, perché già erano stati espulsi nel XVI secolo, in seguito al decreto dei Re Cattolici del 31 marzo 1492. Tra le atrocità del franchismo non si può dunque annoverare la persecuzione degli ebrei. Ne è risultato, in Spagna, un distanziamento nei confronti di un segmento importante della Storia, che continua oggi con il caso di Israele e che il giornalista esprime attraverso la metafora di Micòl e del destino dei Finzi-Contini. Scrive Juliana:

La società spagnola, a differenza di quanto accade in altri paesi europei, non ha mai provato un senso di colpa per l'olocausto. Non si è mai domandata dove sia finita Micòl. Non si è mai domandata perché non c'è più nessuno nel giardino dei FinziContini. 

una vittima che diventa una ugenerazione intera» di vittime ${ }^{40}$, Saladrigas sostiene che il microcosmo ferrarese racchiude una vita intera svelata nella sua più integrale drammaticità ${ }^{41}$; Gándara presenta l'opera di Bassani come un prodotto artistico vincolato da specifiche coordinate spazio-temporali, ma che riesce ad andare ben oltre l'affresco storico o di costume in virtù di un sentimento d'istantaneità dell'hic et nunc al di fuori del quale il tempo è impensabile. Come conseguenza «la Storia, tutti i passati, e il Futuro $»^{42}$ finiscono col concentrarsi nell'istante di mondo ricreato dall'autore. Gándara si sofferma a questo proposito sulla capacità di Bassani di penetrare criticamente una realtà trascendendola, senza bisogno di una diagnosi sociologica, e prestando invece attenzione alle «pieghe del cuore umano». Lo scrittore italiano 
trascende il localismo ed «elude la diagnosi sociale o politica, la critica di costume o di cultura», mostrando interesse ed empatia verso i suoi personaggi.

Con questa definizione, Gándara sembra rivendicare una qualità tutta bassaniana, che per anni era rimasta in ombra, e cioè la straordinaria capacità di carpire le sfumature di un determinato ambiente in un determinato momento storico, penetrando la psicologia dei soggetti che operano in esso. In linea con la posizione di Gándara, José María Guelbenzu, in un articolo del 2007, sgancia definitivamente Bassani da una prima erronea assimilazione al neorealismo, e sottolinea invece la sua attenzione al dettaglio, e la sua scelta di una scrittura descrittiva e lineare che supera i limiti del costumbrismo, al quale più volte si erano arrestati certi esponenti del socialrealismo spagnolo. Così Guelbenzu:

Bassani dovrebbe essere incluso nel neorealismo italiano per ragioni cronologiche, ma non per la sua scrittura, che possiede una magia [encanto] estranea a quel genere. Quando parlo di magia [...] mi riferisco [...] a una scrittura tersa, bella, apparentemente uniforme e dalle potenti correnti occulte, sostenuta dal dettaglio senza essere costumbrista, che dispone personaggi, conflitti e scenari in modo lineare e apparentemente leggero sotto il quale palpitano vite di affascinante intensità [...]. Il mot juste flaubertiano si indovina dietro ogni frase [...] in cui la descrizione, sia fisica che psicologica, che si snoda in subordinate snelle, non eccessivamente cariche ma complesse, si espande in ondate soavi e delicate molto precise, di un sereno vigore che creano un ritmo particolare di grande vivacità espressiva ${ }^{43}$.

Nel 2014 Manuel Hidalgo annuncia in un articolo di «El Mundo» il progetto editoriale di Acantilado di pubblicare separatamente i libri che compongono Il Romanzo di Ferrara. Il primo della serie sarà proprio il primo libro del Romanzo di Ferrara, Dentro le mura (Intramuros). Sebbene collochi Bassani in un'età dell'oro della letteratura italiana, Hidalgo considera l'autore come un «verso suelto», una personalità originale, proprio in virtù di quella sua scrittura «colta, raffinata e linda, che procura grandi soddisfazioni estetiche nel momento stesso in cui affronta politicamente il presente e la Storia drammatica del suo paese $»^{44}$.

Nel 2015 Acantilado dà alle stampe il secondo libro del Romanzo di Ferrara, Gli occhiali d'oro (Las gafas de oro). Questi primi due volumi della saga ferrarese riscuotono un enorme successo. L'opera di Bassani viene descritta come «magistrale», «straordinaria». La scrittrice e critica letteraria Mercedes Monmany parla di un autore tra $\mathrm{i}$ «meno favoriti editorialmente e più ingiustamente dimenticati», che ha dato vita a uno dei «cicli romanzeschi più importanti del XX secolo»" ${ }^{45}$. La critica è compatta nel riconoscere a Bassani le qualità di uno scrittore raffinato, archeologo sensibile di un mondo eterogeneo e conflittuale, che ne porta a galla le contraddizioni in tutta la loro complessità.

36 Ciò che piace di Bassani è la sua capacità di conciliare nella sua scrittura due forti impulsi narrativi della Spagna post-franchista: realismo e psicologismo. Lo scrittore italiano mette in valore il dettaglio senza perdere di vista le sfumature, traccia un contorno che ogni suo personaggio è libero di eludere o ridefinire.

In occasione della pubblicazione de Las gafas de oro edito da Acantilado, il poeta Narcís Comadira, traduttore de L'Airone (La garza) per Seix Barral nel 1970, afferma che la grandezza di Bassani risiede nell'essenza emblematica dei personaggi e delle cose che «formano parte di una Storia immutabile, la nostra, sempre assorta nella sua atrocità $»^{46}$. La Storia, l'io del narratore e quello dei protagonisti parlano all'unisono in 
Bassani e testimoniano di una realtà specifica che si trascende continuamente. Storicismo e psicologismo si incontrano, facendo dell'opera dello scrittore ferrarese ciò che Corradino Vega definisce una «intensificación» ${ }^{47}$ della condizione umana.

\section{A mo' di conclusione}

Come si è visto, la ricezione di Bassani nella Spagna della Transizione si concentra in alcuni segmenti temporali particolarmente densi, in cui la critica diventa più ricettiva, supportata anche dalla politica editoriale delle principali case editrici spagnole. Se si esclude la ristampa del Giardino in edizione tascabile nel 1976, il primo segmento temporale copre gli anni '80 quando, dopo un primo momento di disorientamento culturale, successivo alla morte di Franco, si può parlare di una vera e propria riscoperta di Bassani in Spagna, sia nel mondo editoriale sia da parte della critica specializzata.

Contro ogni previsione inaugura il decennio una raccolta di poesie del 1983, dal titolo generico Poemas, tradotte da Juan Ramos per i tipi di Pablo Lluch. L'assenza di recensioni e di articoli critici induce a pensare che il volume non abbia avuto un particolare successo di pubblico e critica. Maggiore interesse suscitano le due prime edizioni della Novela de Ferrara, quella di Bruguera nel 1984, e quella di Lumen (1989). Nel 1985 escono Epitafio (Lumen) e El jardín de los Finzi-Contini (Seix Barral).

Negli anni '90, nonostante un parziale disinteresse della critica, il Giardino è ristampato da Espasa-Calpe (1993), Seix-Barral (1996) e Planeta (1997). Nel 1995 esce una nuova edizione de La garza (L'airone) per i tipi di Cátedra.

41 A partire dagli anni zero l'attenzione critica a Bassani è costante. In particolare, nei primi tre anni del secolo, dopo la morte dell'autore, si tenta un primo e parziale bilancio della sua traiettoria poetica. Tra il 2001 e il 2004 è ristampato ben tre volte il Giardino, edito rispettivamente da Círculo de Lectores, El País ed Espasa-Calpe. Sarà ancora questione di Bassani nel 2007, quando la Novela de Ferrara è ristampata da Lumen. Nello stesso anno Tusquets Editores pubblica una nuova edizione del Jardín de los Finzi-Contini. Infine, dopo una nuova riedizione della Novela de Ferrara da parte della Casa Debolsillo nel 2009, arriviamo al biennio 2014-2015, quando la critica mostra un rinnovato interesse per Bassani, in coincidenza con il progetto editoriale di Acantilado, che decide di dare alle stampe i singoli volumi del Romanzo di Ferrara, iniziando proprio da Dentro le mura (Intramuros).

Il successo delle opere di Bassani nella Spagna democratica non è stato eccezionale dal punto di vista delle cifre. Carlos Barral aveva ragione quando già negli anni '60 scriveva: «Siamo molto interessati al Bassani anche se non crediamo troppo probabile che diventi un best-seller come pare sia accaduto in Italia» ${ }^{48}$. Ciò si può spiegare col fatto che l'autore italiano è sempre stato accolto da una minoranza interessata a un prodotto di qualità, fra cui si contano non pochi intellettuali, poeti e narratori.

A titolo di esempio possiamo addurre il professore e scrittore di nicchia Eduardo Alonso che nel 1992 pubblica un romanzo breve ${ }^{49}$ in cui racconta le vicende di un professore di storia in preda a una crisi affettiva e che diventa ossessionato dalla Micòl di Bassani. L'eleganza dello stile di Alonso, in cui si avverte una lontana eco bassaniana, ha indotto la critica a applicare al suo romanzo l'etichetta di «novela lírica» ${ }^{50}$. Ogni parola è ben calibrata all'interno di una costruzione romanzesca in cui Alonso mantiene un delicato 
equilibrio tra realtà e finzione. L'attrazione che il protagonista prova per Micòl apre lo spazio di una sorta di metanarrazione in cui si intersecano sezioni narrative di diverso livello. In seguito al divorzio da sua moglie, il protagonista si abbandona rapito alla lettura del Giardino dei Finzi-Contini e ne è indotto a intraprendere un viaggio a Bologna e Ferrara sulle tracce di Micòl. A Bologna incontra Chiara, una ragazza che viene a svolgere la 'funzione Micòl' nella vita del professore. Scrive Alonso:

Le mie fantasie erano iniziate alcuni mesi prima, poco dopo essermi separato da Ana, quando sognavo un incontro impossibile. Leggevo allora un romanzo di Bassani [...]. Letto un paragrafo mi perdevo per le quiete strade di una città di provincia [...]. L'unica cosa certa è che finii per essere ossessionato dalla protagonista di quel romanzo malinconico. Si chiamava Micòl Finzi-Contini, era smilza, e doveva trovarsi da qualche parte, però chissà che aspetto aveva adesso, chissà com'erano i suoi occhi, che cosa avrebbe pensato se mi avesse veduto. [...] Micòl era una di quelle donne destinate ad amare senza scrupoli, ed io, al contrario, sono sempre stato propenso a compensare le mie goffaggini in amore con fantasie solitarie. [...] In sogno la vedevo tornare in bicicletta dal campo da tennis, la spiavo nella sinagoga di via Mazzini [...] le dicevo addio quando se ne andava in vacanza a Comacchio sulla Citroën nera della famiglia. [...] Diventavo tanto diverso, via via che mi addentravo nel libro e diventava tanto reale la Micòl delle mie fantasie, che quella ferrarese del romanzo mi sembrava un'impostora, o almeno un ritratto impreciso di quella autentica, mentre la vera Micòl, nonostante avesse un altro nome, doveva starmi ad aspettare da qualche parte ${ }^{51}$.

Chiudiamo con qualche considerazione sulle case editrici che hanno determinato la fortuna editoriale di Bassani. Eccetto un'edizione de Il giardino dei Finzi-Contini pubblicata dal quotidiano «El País» nel 2003 - e che può essere considerata come un primo tentativo di 'popolarizzare' l'opera di un autore apprezzato prevalentemente da un pubblico colto ed elitario - è rilevante il fatto che Bassani sia sempre stato pubblicato da case editrici importanti, di ottima qualità, mosse dalla precisa intenzione di riorientare culturamente il pubblico. Da questo punto di vista, non è forse un caso che Planeta, la casa editrice di tendenza «divulgativo/comercial»" ${ }^{52}$ che più interpreta lo spirito effervescente del tempo con un catalogo tra i meno esigenti e più popolari, pubblichi solo un'opera del ferrarese, Il giardino dei Finzi-Contini, nel 1997. Il resto della produzione bassaniana è stato pubblicato in collane che puntano più all'eccellenza del prodotto che alla tiratura. È il caso di Espasa-Calpe, Círculo de Lectores ed Ediciones Cátedra - presso cui esce L'airone (La garza) nel 1995 - Casa che nel 1997 ha ricevuto dal Ministerio de Educación y Cultura il Premio Nacional per la «mejor labor editorial y cultural». È il caso di Bruguera, la cui pubblicazione del Romanzo di Ferrara si spiega con un'evoluzione interna della casa editrice. Negli anni del franchismo, Bruguera era nota per la sua diffusione di narrativa popolare spagnola e fumetti, e aveva una collana di tascabili che tra gli anni ' 60 e ' 70 diede alle stampe una letteratura di intrattenimento non troppo esigente. Intorno agli anni '70, l'arrivo in redazione dell'argentino Ricardo Rodrigo cambiò l'impostazione della casa editrice e fece di Bruguera un marchio di riferimento nella pubblicazione di grandi opere letterarie. Così, insieme a Scott Fitzgerald, Truman Capote, Italo Calvino, John le Carré e Jorge Luís Borges, a metà degli anni ' 80 entra nel catalogo l'opera di Bassani. Fu un'operazione guidata dalla volontà di recupero e di riscatto di grandi figure della letteratura mondiale e fece di Bruguera il fulcro dell'editoria spagnola dell'epoca. In modo simile, la pubblicazione del Romanzo di Ferrara presso Lumen ne rispecchia coerentemente l'ambizioso programma editoriale. Dagli anni '60 e sotto la direzione di Esther Tusquets la casa barcellonese sviluppa un 
catalogo di riconosciuto prestigio culturale. Lumen ha anche dato alle stampe l'unica raccolta di poesie di Bassani che sia stata finora pubblicata in Spagna.

A conferma del carattere elitario della ricezione spagnola dello scrittore ferrarese, proprio l'editrice Esther Tusquets ha dichiarato nel 2009, in un'intervista rilasciata a Carles Geli: «Potrei dire Joyce o Woolf, ma oggi sono orgogliosa di aver pubblicato Giorgio Bassani» $\aleph^{53}$.

Sottolineiamo infine che nonostante il carattere elitario della ricezione di Bassani in Spagna, l'interesse per questo scrittore non è mai venuto meno, e la critica specialistica non ha mai cessato di riflettere sulla sua opera. Sembra dunque giustificata l'etichetta «clásico tímido» proposta dallo scrittore e critico Alejandro Gándara, che con l'aggettivo 'timido' designa un autore dal successo editoriale contenuto ma costante. Se poi consideriamo che l'interesse della critica bassaniana è stato in gran parte determinato da temi come quello della responsabilità, dell'impegno civile e del dovere di memoria, sembra legittimo parlare - almeno per quanto riguarda la penisola iberica - di un «clásico tímido» ma «esencial» $»^{54}$.

\section{ALLEGATO}

\section{Edizioni di opere di Giorgio Bassani in spagnolo prima del 1975}

Poetas italianos de hoy. Giorgio Caproni, Vittorio Sereni, Vittorio Bodini, Giorgio Bassani, Barcellona, «Entregas de poesía», n² 24, 1950 (rivista diretta da Juan Ramón Masoliver).

El jardín de los Finzi-Contini, Barcellona, SeixBarral, 1963 (trad. di Juan Petit).

Historias de Ferrara, Barcellona, Seix Barral, 1967 (trad. di Elena Sáez de Diamante e Juan Augustín Goytisolo).

El jardín de los Finzi-Contini, Barcellona, Seix Barral, 1967 (trad. di Juan Petit).

El jardín de los Finzi-Contini, Barcellona, Seix Barral-Biblioteca de Bolsillo, 1968 (trad. di Juan Petit).

Detrás de la puerta, Barcellona, Seix Barral, 1969 (trad. di Sergio Pitol).

La garza, Barcellona, Seix Barral, 1970 (trad. di Narcís Comadira).

Lida Mantovani y otras historias de Ferrara, Barcellona, Barral Editores, 1971 (trad. di Sergio Pitol).

Los anteojos de oro, Barcellona, Barral Editores, 1972 (trad. di Sergio Pitol).

El jardín de los Finzi-Contini, Barcellona, Seix Barral, 1973 (trad. di Juan Petit).

El olor del heno, Barcellona, Seix Barral, 1974 (trad. di Carlos Manzano).

\section{Edizioni di opere di Giorgio Bassani in spagnolo dopo il 1975}

El jardín de los Finzi-Contini, Barcellona, Seix Barral, 1976 (trad. di Juan Petit). 
Poemas en Quervo, Cuadernos de Cultura, Valencia, Pablo Lluch, 1983 (trad. di Juan Ramos).

La novela de Ferrara, Barcellona, Bruguera, 1984 (trad. di Carlos Manzano).

Epitafio, Barcellona, Lumen, 1985 (trad. di Carlos Manzano).

El jardín de los Finzi-Contini, Barcellona, Seix Barral, 1985 (trad. di Juan Petit).

La novela de Ferrara, Barcellona, Lumen, 1989 (trad. di Carlos Manzano).

El jardín de los Finzi-Contini, Barcellona, Espasa-Calpe, 1993 (trad. di Carlos Manzano, prólogo di Antonio Colinas).

La garza, Madrid, Cátedra, 1995 (a cura di Anna Dolfi, trad. di Carlos Manzano).

El jardín de los Finzi-Contini, Barcellona, Seix Barral, 1996 (trad. di Juan Petit).

El jardín de los Finzi-Contini, Barcellona, Planeta, 1997 (trad. di Carlos Manzano).

El jardín de los Finzi-Contini, Barcellona, Círculo de lectores, 2001 (trad. di Carlos Manzano).

El jardín de los Finzi-Contini, Madrid, El País, 2003 (trad. di Carlos Manzano).

El jardín de los Finzi-Contini, Barcellona, Espasa Calpe, 2004 (trad. di Carlos Manzano, prólogo di Antonio Colinas).

El jardín de los Finzi-Contini, Barcellona, Editorial Tusquets, 2007 (trad. di Carlos

Manzano).

La novela de Ferrara, Barcellona, Lumen, 2007 (trad. di Carlos Manzano).

La novela de Ferrara, Barcellona, Debolsillo, 2009 (trad. di Carlos Manzano, prólogo di Pier Paolo Pasolini).

Intramuros, Barcellona, Acantilado, 2014 (trad. di Juan Antonio Méndez).

Las gafas de oro, Barcellona, Acantilado, 2015 (trad. di Juan Antonio Méndez).

Edizioni di opere di Giorgio Bassani in catalano prima del 1975

Darrera la porta, Barcellona, Edicions 62, 1968 (trad. di Manuel Carbonell).

Edizioni di opere di Giorgio Bassani in catalano dopo il 1975

Darrera la porta, Barcellona, Edicions 62, 1989 (trad. di Manuel Carbonell).

Dins la muralla, Barcellona, Edicions 62, 1989 (trad. di Joan Casas).

Epitafi, Alzira, Bromera, 1996 (trad. di Josep Ballester).

El jardí dels Finzi-Contini, Barcellona, Proa, 1987 (trad. di Carme Serrallonga).

El jardí dels Finzi-Contini, Barcellona, GE, 1996 (trad. di Carme Serrallonga).

El jardí dels Finzi-Contini, Barcellona, Proa, 2007 (trad. di Carme Serrallonga).

Edizioni di opere di Giorgio Bassani in basco (euskara) dopo il 1975

Finzi-Continitarren lorategia, Euba, Ibaizabal, 1997 (trad. di Koldo Biguri). 


\section{NOTE}

1. Preceduta soltanto dalla traduzione di alcune poesie antologizzate in un volume curato da J. R. Masoliver: Poetas italianos de hoy. Giorgio Caproni, Vittorio Sereni, Vittorio Bodini, Giorgio Bassani, «Entregas de poesía», n² 24, 1950.

2. Così già scrive Barral a Linder nel giugno del 1962: "Ti prego non fare niente con un altro editore di lingua spagnola. Siamo molto interessati al Bassani» (Fondazione Arnoldo e Alberto Mondadori, Milano, Agenzia letteraria internazionale - Erich Linder, 1962, B. 22, fasc. 13 [Seix Barral], Carlos Barral a Erich Linder, Barcellona, 19 giugno 1962, dattiloscritto originale).

3. Ringrazio Andrea Bresadola per avermi permesso di utilizzare i risultati della sua ricerca che è attualmente ancora in corso di stampa.

4. Dirección General de Información, « $\mathrm{ABC}$ », 9 gennaio 1963.

5. Il bisticcio è di Carlos Barral, che così scrive nelle sue Memorias: «Milano era allora, anche se non esattamente la capitale letteraria d'Europa, la capitale dell'ingegno e dell'inventiva editoriale dalle alte ambizioni, una vera e propria corte nelle vicinanze del palazzo reale, che si trovavava a Torino, dove regnava Einaudi circondato dai suoi pretoriani 'einauditi'» (C. Barral, Memorias (Los años sin excusa), Barcellona, Lumen, 2015, p. 664. Delle memorie di Barral esiste una versione italiana parziale: Carlos Barral. Il volo oscuro del tempo. Memorie di un editore poeta. 1936-1987, a cura di R. Baravalle, P. Collo e G. Felici, Milano, Il Saggiatore, 2011). Preciso che qui e in tutto il saggio sono mie le traduzioni dallo spagnolo.

6. C. Barral, Memorias (Años de penitencia), cit., p. 560.

7. C. Barral, Carta de Seix Barral a los lectores de La Vanguardia, «La Vanguardia», 6 novembre 1963.

8. J.R. Masoliver, «... Soli rimasti dei giardini sepolti». Nuestra interior estela funeraria, «La Vanguardia», 4 dicembre 1963.

9. Negli anni '70 Pérez Minik include Bassani tra gli autori rappresentativi di un nuovo «circuito democrático» che va da Vittorini a Arpino. Lo studio di Minik analizza il 'nuovo' panorama letterario italiano, e si sofferma in particolare su quei romanzi che gli sembrano corrispondere ai bisogni della Spagna di allora. Il critico mette su uno stesso piano non solo Vittorini, Pavese e Calvino ma anche Pratolini, Cassola, Piovene, Gadda e Bassani, tutti autori che gli sembrano poter nutrire il tessuto narrativo spagnolo, pur proponendo, ciascuno di loro, una diversa poetica realista, e una diversa morale. Giorgio Bassani viene introdotto come un intellettuale che deve essere collocato in una posizione intermedia tra la generazione postbellica e la nuova generazione, guidata da figure come Calvino, Cassola e Pasolini (D. Pérez Minik, Un circuito democrático: de Elio Vittorini a Giovanni Arpino, in Id., La novela extranjera en España, Madrid, Talleres de Edición Josefina Betancor, 1973, p. 229).

10. La novela social che si afferma in Spagna intorno agli anni Cinquanta riflette le principali preoccupazioni sociali dell'epoca trattando temi quali lo sfruttamento del proletariato e la condizione contadina, industriale e borghese, sviluppati senza uscire dai limiti di una narrazione stereotipata. I personaggi si presentano come caratteri tipici che si muovono all'interno di un contesto nazionale e regionale specifico, descritto con abbondanza di particolari. Sull'argomento vedi: P. Gil Casado, La novela social española (1920-1971), Barcellona, Seix-Barral, 1973; S. Sanz Villanueva, Historia de la novela social española (1942-1975), Granada, Editorial Alhambra, 1986; F. Alamo Felices, La novela social española: conformación ideológica, teoría y crítica, Almería, Universidad de Almería, 1996.

11. Letraherido è un gallicismo (lettre ferit) usato da Montaigne in Du pédantisme, e introdotto in catalano nell'accezione molto ampia di 'amante delle lettere'. Il termine si afferma in Catalogna negli anni '50, soprattutto fra i membri dalla celebre Escuela de Barcelona.

12. Come noto, infatti, anche Bassani ha sempre «mantenuto un atteggiamento di grande perplessità» nei confronti del neorealismo, per quel suo «sentimentalismo populistico», per «la facilità con cui $[. .$.$] si metteva al servizio della pratica, cioè della politica». La sua più volte$ 
dichiarata necessità di un'arte capace di riflettere la realtà in tutte le sue contraddizioni sembra trovare palesi affinità nell'atteggiamento critico della classe intellettuale spagnola che alla fine degli anni'60 inizia ad interrogarsi con un certo scetticismo sulla reale efficacia di una letteratura socialista.

13. In genere il termine designa in modo specifico il periodo compreso fra la morte di Franco (20/11/1975) e l'approvazione della Costituzione spagnola (29/12/1978), ma può essere ampliato fino ad includere gli inizi degli anni '90 quando, con il trattato di Maastricht, la Spagna entra ufficialmente nello spazio europeo.

14. Cfr. P. Rodríguez Pacheco, La otra mirada. Literatura española ¿crimen o suicidio?, Barcellona, Editorial Carena, 2015, p. 206.

15. Barral editore «exquisito» è un'etichetta di J. A. Molina Foix che compare nel libro di S. VilaSanjuán, Pasando página: autores y editores en la España democrática, Barcelona, Destino, 2003, p. 101. 16. Cfr. J. Gracia, La resistencia silenciosa. Fascismo y cultura en España, Barcelona, Anagrama, 2014.

17. J. F. Beaumont, Giorgio Bassani. «El jardín de los Finzi-Contini» es una toma de posición contra el fascismo, «El País», 27 maggio 1981.

18. Il quotidiano nazionale «El País» è stata una della principali fonti di questa ricerca. Negli anni in cui avviene la ricezione di Bassani in Spagna, il giornale è uno dei mezzi di orientamento culturale più importanti della penisola iberica, e vi hanno trovato spazio le voci più autorevoli della critica e della letteratura spagnola.

19. È stata inoltrata una richiesta agli archivi di RTVE per recuperare la registrazione del programma con l'intervista allo scrittore.

20. A. Colinas, Giorgio Bassani: «vivimos el mundo trágico del industrialismo», «El País», 10 gennaio 1983.

21. A. Munné, Giorgio Bassani considera que los artistas son muertos que tratan de regresar a la vida a través de la muerte, «El País», 18 settembre 1985.

22. J. Torán in Giorgio Bassani o el compromiso con la memoria europea («Ahora», 10 ottobre 2016) parla a questo proposito di «evidente fiuto letterario».

23. R. Saladrigas, Giorgio Bassani, hijo y recreador de Ferrara, «La Vanguardia», 30 maggio 1985.

24. G. Bassani, La novela de Ferrara, trad. di C. Manzano, Barcellona, Bruguera, 1984.

25. Il costumbrismo in letteratura è un movimento sviluppatosi in Spagna nel XIX secolo, e caratterizzato dalla descrizione oggettiva di usi e costumi di un determinato ambiente, popolo o classe sociale. Cfr. R. Espejo-Saavedra, Autenticidad y artificio en el costumbrismo español, Madrid, Ediciones de la Torre, 2015.

26. La nozione di 'amnesia storica' è stata introdotta da J. M. Izquierdo in Narradores españoles novísimos de los años '90, «Revista de estudios hispánicos», vol. XXV, $\mathrm{n}^{\circ} 2,2001$. Izquierdo chiarisce anche la differenza tra la generazione poetica dei novísimos - proposti da J. M. Castellet nell'antologia Nueve novísimos poetas españoles, Barcellona, Barral Editores, 1970 - e i narradores novísimos della nuova narrativa joven degli anni ' 90.

27. Annunciando la scomparsa di Bassani, la giornalista culturale Lola Galán lo definisce come «uno de los autores más representativos de la literatura italiana de la segunda mitad de siglo XX». Ripercorrendo la sua traiettoria poetica, essa sottolinea il suo impegno e il suo profondo senso di responsabilità sociale e politica, dall'antifascismo giovanile a Italia Nostra (L. Galán, Fallece en Roma Giorgio Bassani, autor de «El jardín de los Finzi-Contini». El escritor, de 84 años, padecía Alzheimer, «El País», 14 aprile 2000). Degni di nota anche due saggi pubblicati su «El País» il 18 dicembre dello stesso anno: L. M. Diez, El fermento de la memoria e A. Padilla, La memoria superviviente.

28. A. Gándara, Un clásico tímido, «El Mundo», 15 novembre 2007.

29. J. A. Maydeu, La novela de Ferrara, de Giorgio Bassani, «Letras Libres», 31 dicembre 2007.

30. G. M. Goloboff, La ciudad íntima de Giorgio Bassani, "Quimera: Revista de literatura», n 196, 2000, p. 55. Intervenendo a sua volta, e più recentemente, su Ferrara, Alejandro Gándara parla di 
"pérdida del alma», di smarrimento dei valori nella città pre e post-fascista; secondo lo scrittore con le leggi razziali si è perso lo spirito del secolo (A. Gándara, La pérdida del alma, «El Mundo», 13 gennaio 2015).

31. L. M. Díez, El fermento de la memoria, cit.

32. J. Torán, Giorgio Bassani o el compromiso con la memoria europea, cit.

33. E. Juliana, España e Israel: ¿Dónde está Micol?, «La Vanguardia», 9 gennaio 2009.

34. J. M. Herrera Pérez, Giorgio Bassani y la memoria histórica, "Cuadernos hispanoamericanos», $\mathrm{n}^{\circ}$ 791, 2016, pp. 120-129.

35. P. Marín, Giorgio Bassani. La novela de una vida, «Quimera: Revista de literatura», $\mathrm{n}^{\circ} 101,1990$, pp. 34-37.

36. "Quanto a me, ho scelto mezzo secolo fa da che parte stare. Mi sono difeso dalla identificazione di me stesso come ebreo, entrando nell'antifascismo militante clandestino, al seguito di un uomo che si chiamava Carlo Ludovico Ragghianti. [...] Militante coraggioso, Ragghianti era culturalmente uno storicista, un seguace di Croce» (N. Ajello, "Un giorno sì e uno no gioco a tennis", «Millelibri», $n^{\circ}$ 5, 1988, p. 72).

37. G. Bassani, Le leggi razziali, in Opere, a cura di R. Cotroneo, Milano, Mondadori, 2009, p. 1438.

38. V. Jankélévitch, La coscienza ebraica, Firenze, Giuntina, 1995, p. 87.

39. P. Marín, Giorgio Bassani. La novela de una vida, cit., p. 37.

40. J. Torán, Giorgio Bassani o il compromiso con la memoria europea, cit.

41. R. Saladrigas, Un microcosmo que encierra una vida entera, «El País», 1 dicembre 2007.

42. A. Gándara, La pérdida del alma, «El Mundo», 13 gennaio 2015.

43. J. M. Guelbenzu, Un microcosmo que encierra una vida entera, «El País», 1 dicembre 2007.

44. M. Hidalgo, El jardinero y el infierno, «El Mundo», 6 dicembre 2014.

45. M. Monmany, Giorgio Bassani a los pies de Ferrara, «ABC», 27 dicembre 2014.

46. N. Comadira, Bassani, «Ara», 6 maggio 2016.

47. C. Vega, Verdad y belleza, «Estado crítico», 2 settembre 2015.

48. Fondazione Arnoldo e Alberto Mondadori, Milano, Agenzia letteraria internazionale - Erich Linder, 1962, B. 22, fasc. 13 (Seix Barral), Carlos Barral a Erich Linder, Barcellona, 19 giugno 1962, dattiloscritto originale.

49. E. Alonso, El retrato del Schifanoia, Barcellona, Muchnik Editores, 1992.

50. A. Basanta, El retrato del Schifanoia, «ABC», 26 giugno 1992.

51. E. Alonso, El retrato del Schifanoia, cit., pp. 10-11.

52. R. Acín, Narrativa o Consumo Literario (1975-1987), Zaragozza, Prensas Universitarias de Zaragoza, 1990, p. 83.

53. C. Geli, A mi edad, uno se lo permite todo, «El País», 11 novembre 2009.

54. A. Gándara, Un clásico tímido, «El Mundo», 15 novembre 2007.

\section{RIASSUNTI}

La fortuna editoriale di Giorgio Bassani in Spagna va studiata tenendo conto di una circostanza storica, quella del franchismo, che fa da vero e proprio spartiacque alla politica culturale del Paese. La ricezione del ferrarese nella penisola iberica inizia in un periodo di urgenza culturale e intellettuale sotto il regime di Franco e continua negli anni della Transizione e della democrazia, mostrando un'evoluzione critica interna direttamente legata alle esigenze politico-culturali della 
Spagna. Attraverso i concetti di estetica letteraria, memoria, testimonianza e responsabilità della scrittura, il saggio analizza la 'funzione Bassani' in Spagna dalla Transizione democratica a oggi.

La fortuna editorial de Giorgio Bassani en España tiene que investigarse a raíz del franquismo, o sea una anomalía histórica que ha constituido un hito en la política cultural del país. La recepción del escritor italiano en la península ibérica, arranca en un momento de urgencia cultural e intelectual bajo el régimen de Franco y continúa en los años de la Transición y democracia, cuando es evidente una evolución crítica interna directamente vinculada a las exigencias políticas y culturales de España. A través de los conceptos de estética literaria, memoria, testimonio y responsabilidad de la escritura, el ensayo precisa investigar la «función Bassani» en España, desde la Transición democrática hasta hoy.

Le succès de Giorgio Bassani en Espagne doit être étudié en tenant compte de l'anomalie historique du franquisme qui a marqué une véritable fracture dans la politique culturelle du pays. L'accueil de Bassani en Espagne commence dans un période d'urgence culturelle et intellectuelle sous le régime de Franco et continue dans les années de la transition et de la démocratie en montrant une évolution critique intérieure liée aux exigences politiques et culturelles de l'Espagne. À travers les concepts d'esthétique littéraire, de mémoire, de témoignage et de responsabilité de l'écriture, l'étude explore la «fonction Bassani » en Espagne de la transition démocratique à aujourd'hui.

\section{INDICE}

Mots-clés : esthétique littéraire, mémoire, témoignage, responsabilité de l'écriture

Parole chiave : estetica letteraria, memoria, testimonianza, responsabilità della scrittura

Palabras claves : estética literaria, memoria, testimonio, responsabilidad de la escritura

\section{AUTORE}

\section{SILVIA DATTERONI}

Universidad de Granada e Sapienza Università di Roma 